\title{
Position control of robot manipulator by torque equillibrium method
}

\author{
Mayur Pateliya ${ }^{1^{*}}$ and Jyoti Ohri ${ }^{2}$ \\ M.Tech Scholar, Department of Electrical Engineering, National Institute of Technology, Kurukshetra, Haryana, \\ India ${ }^{1}$ \\ Professor, Department of Electrical Engineering, National Institute of Technology, Kurukshetra, Haryana, India ${ }^{2}$
}

(C2016 ACCENTS

\begin{abstract}
This paper presents position control of a two-link manipulator arm using a control technique employed only towards motor-side. Here the coupling effect between the motor and manipulator dynamics is considered as a disturbance signal. Proportional-integral-derivative (PID) (tuned by genetic algorithm (GA)) is designed to control the disturbances with some tolerance limit. Simulation results are presented which shows performance comparison of responses with and without disturbance signal.
\end{abstract}

\section{Keywords}

Two-link arm, PMDC motor, Disturbance signals, Coupling, Classical PID, GA tuned PID, ITAE.

\section{Introduction}

As the application area of manipulator is very wide and should be precisely accurate, control has the prime role. There are specifically two areas where control is required, first is movement of the arm and second is the force at the time of interaction with operating surface [1]. This paper is focused on the movement/position control of manipulator. Controlling techniques are used according to the types of motion, as in point to point motion no need to take care of the path of motion whereas in guarded motion the path of motion plays a key role. Designing a trajectory for the movement of manipulator becomes important here.

With recent advances in technology, in spite of new progress on control area, PID type controllers are undoubtedly the most commonly used controllers in the industry due to their practicality [2-6]. Their relatively simple structures that can be easily implemented and the availability of well-defined rules for the tuning of controller parameters are the main reasons for making them preferable in real-time applications. But there are some weaknesses in the classical PID control [4], such as noise degradation in the derivative control, loss of performance in the control law in the form of a linear weighted sum and complications bought by the integral control.

*Author for correspondence

211
In order to enhance the performance of PID, many combinations are being developed. Such as tuning of PID parameters with linear-quadratic regulator (LQR) [2], fractional order PID [7], implementation of fuzzy logics for tuning of PID [3], neuro-fuzzy for PID [6], tuning of PID parameters using genetic algorithms (GA) [8, 9]. GA is used to find the best possible parameters of PID. This paper mainly concentrates on the controlling motor-side dynamics only. Thus, allowing link-side to interact freely with the operational environment. Principle behind this is to generate equilibrium torque by actuator so that it can give desired speed and accelerations to the link to track the position accurately.

Paper further organized into sections as follows: Section II gives brief theory of the approach developed for the technique, Section III contains Controlling strategy, and simulation results are illustrated in section IV, followed by the concluding remark in section $\mathrm{V}$.

\section{Approach}

First, an idea for independent motor-side control was coined out from the dynamic equations of both actuator and link. As both the dynamics are related to each other by torques, or other way to say this is, they are related by angular positions. So what if controlled torque (or position/velocity) is given to the link so as it can track the desired trajectories? Answer is here in the dynamic equations itself. 


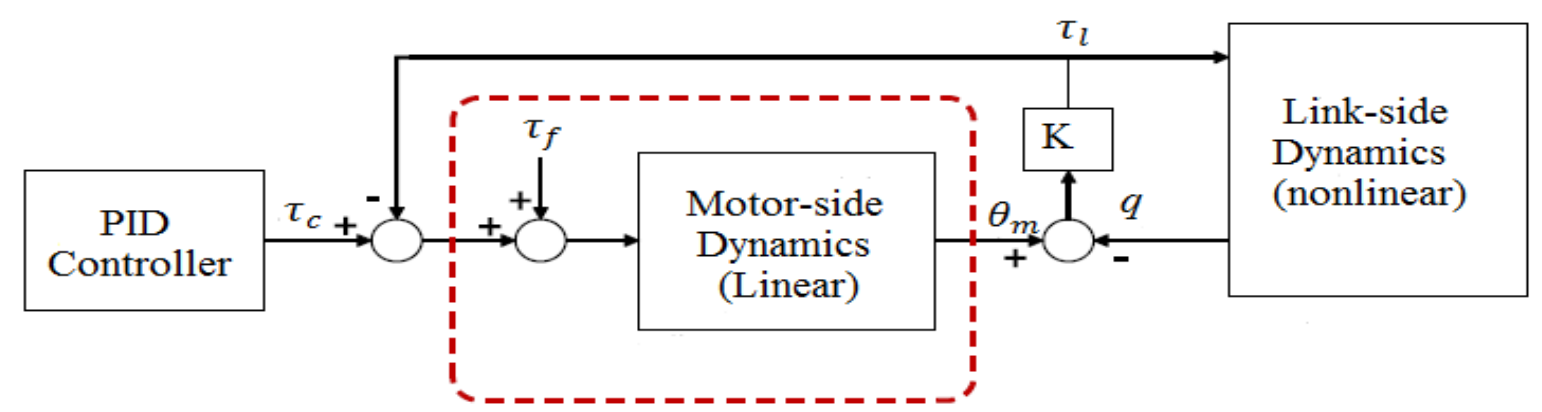

Figure 1 Linear motor-side dynamics works in conjunction with nonlinear link-side dynamics

For the output torque of the actuator, load which is mounted on it (here it is manipulator arm) will cause disturbance or we can say it will oppose the motor torque by virtue of its inertia and other factor as considered in the equation. If we incorporate that oppose (which is nothing but the load torque) in the actuator controlling scheme as extra input torque or as disturbance torque as in Figure 1 and Figure 3, than the controller will control the overall motion of end-effector.

Motor torque generated from the given voltage is say, which is actually affected by so many factors though we ignore friction, environmental disturbances, etc. Angular acceleration given to the load from the motor will generate reverse torque on the shaft by virtue of its inertia that will act as disturbance to the motor torque. Disturbance also caused due to the inertias and damping of the motor shaft itself. So if we incorporated these two disturbances than the torque that is actually transferred to the load is modeled by the equation.

$$
\tau=\tau_{m}-\tau_{l}-B_{m} \frac{d \theta_{m}}{d t}-J_{m} \frac{d^{2} \theta_{m}}{d t^{2}}
$$

From (1), inertia and damping effects of the motor can be considered on the motor torque since they are because of the shaft of motor itself. So (1) becomes

$$
T=\tau+B_{m} \frac{d \theta_{m}}{d t}+J_{m} \frac{d^{2} \theta_{m}}{d t^{2}}=\tau_{m}-\tau_{l}
$$

Now our main aim is to generate the output torque of the motor shaft such that it can transfer net torque $\mathrm{T}$, as in (2), to the load mounted on it. Torque generated by the load on to the shaft due to its virtue of inertia can be calculated from the dynamic modeling of the manipulator arm equations (5).

\section{Modeling}

\subsection{Modeling of actuator}

Modeling of motor describes the relation between the output torque and the controlling input voltage along with the effects of inertia and damping of shaft of motor, as in Figure 2, and effect of load on the motor [5].

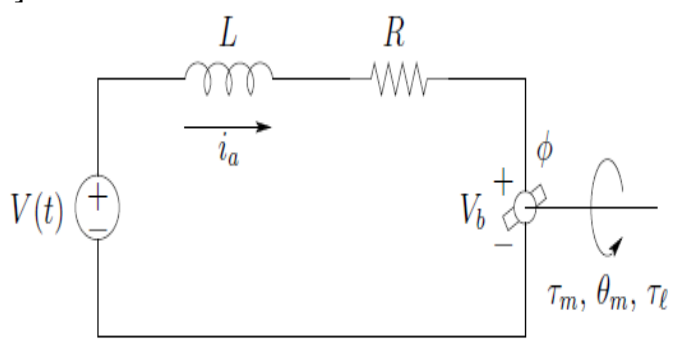

Figure 2 Equivalent and electro-mechanical representation of PMDC motor

Electrical equivalent:

$$
L \frac{d i_{a}}{d t}+R i_{a}=V-V_{b}
$$

Mechanical equivalent:

$$
J_{m} \frac{d^{2} \theta_{m}}{d t^{2}}+B_{m} \frac{d \theta_{m}}{d t}=\tau_{m}-r \tau_{l}
$$

Where, $\mathrm{V}$ is input voltage to actuator, $\mathrm{L}$ is armature inductance $(0.0024 \mathrm{H}), \mathrm{R}$ is armature resistance (4 $\Omega), \quad V_{b}$ is back emf; $i_{a}$ is armature current; $\theta_{m}$ is rotor position (radians); $\tau_{m}$ is generated torque by motor; $\tau_{l}$ is load torque (manipulator arm is a load); $\mathrm{r}$ is gear reduction ratio, $\left(\mathrm{J}_{\mathrm{m}}=4.2 \times 10^{-5} \mathrm{~kg} \cdot \mathrm{m}^{2}, \mathrm{~B}_{\mathrm{m}}=\right.$ 0.01 N.m.sec/rad, $\mathrm{Kv}=\mathrm{K}_{\mathrm{m}}=\mathrm{K}=0.053$ ).

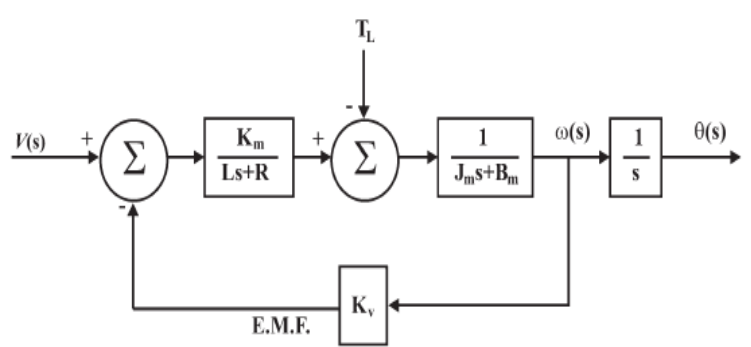

Figure 3 Mathematical model of DC motor in sdomain 


\subsection{Modeling of manipulator}

Dynamic robot models describe the relations between the motions of the robot and the forces that cause the motion. Dynamic model of a robot manipulator can be expressed as

$$
\tau=M(q) \ddot{q}+c(q, \dot{q}) \dot{q}+g(q)+f(\dot{q})
$$

Where, $\tau$ is the actuator torque vector, $M(q)$ is the inertia matrix, $c(q, \dot{q})$ is the vector Coriolis and centripetal torques, $g(q)$ is the gravity torque vector and $f(\dot{q})$ is the vector of, possibly nonlinear, joint friction torques.

In general, for any DOF manipulator arm, dynamic model or equations of motion, those are derived from the Euler-Lagrange method, will always be two degree of linear/non-linear differential equations of the form shown above.

Table 1 Physical parameters of the 2-link of robotic

\begin{tabular}{lll}
\hline Sr.No. & Parameter & Value \\
\hline 1. & Length $(\mathrm{m})$ & $\mathrm{L} 1=0.25, \mathrm{~L} 2=0.15$ \\
2. & Mass $(\mathrm{kg})$ & $\mathrm{M} 1=1.95, \mathrm{M} 2=0.93$ \\
3. & Tensor inertia $\left(\mathrm{kg}-\mathrm{m}^{2}\right)$ & $\mathrm{I} 1=0.0980, \mathrm{I} 2=0.9800$ \\
\hline
\end{tabular}

\section{Controlling strategies}

\subsection{Classical PID}

An optimally tuned PID is integrated in the system in the form of

$$
V(t)=K_{P} e(t)+K_{I} \int e(t) d t+K_{D} \frac{d e(t)}{d t}
$$

Where, $K_{P}, K_{I}$ and $K_{D}$ represents the proportional, integral and derivative gains respectively. $e(t)$ denotes the error in the speed signal. The controller is tuned optimally by using a pattern search optimization method, and the gains are found to be $K_{P}=203, K_{I}=86$ and $K_{D}=48$.

\subsection{GA tunned PID}

Genetic algorithms are global, parallel search and optimization methods founded on Darwinian principle (survival of the fittest). This works with a population of potential solutions to a problem. The population is evolved over generations to get the best possible solution [9]. Figure 4 shows the flow chart of GA to search the PID parameters which satisfies our requirement. In this algorithm first we create the initial population and check that our requirement is satisfied or not. In case it is not satisfied every time a new population is created by using few percent of champions and rest is from crossover of parents.



Figure 4 Flowchart of GA for PID tuning

Initialization of GA is done using the upper bound and lower bound to the values of the PID parameters that was obtained for classical PID [10]. Algorithm for GA developed in the m-file of MATLAB and thereby calling the Simulink function wherein Simulink models are developed for the system. Fitness function defined was according to the performance index. Selection of the performance index is equally important as its minimization. There are several performance indices which include the desired system characteristics and, in addition, have good mathematical tractability. Performance index is the integral of absolute error (IAE) defined by

$$
I A E \cong \int_{0}^{\infty}|e(t)| d t
$$

The index is very reasonable since both the fast but highly oscillatory systems and the sluggish systems will give large IAE value. Minimizing of IAE will 
provide acceptable relative stability and speed of response. And also, a finite value of IAE implies that the steady-state error is zero.

If fitness value is feasible, parameters is added to population along with fitness value otherwise discarded. Once the initial population is created it is sorted best on fitness value in increasing order. If the lowest fitness value satisfy user criteria, process stops there, otherwise new generation is created again.

\section{Simulation results}

Implementing above theory to the Robotics manipulator arm, starting with implementation on basic two-link manipulator arm, whose every joints are independently driven by PMDC motors employed for each. Dynamic modellings for a mathematical equivalent of the actuator and the manipulator is done using Lagrange's Euler method [11]. PMDC motor in a negative feedback system becomes stable and will track the output torque with some tolerable error as shown in Figure 5. Herein actuator is very slow with settling time of $80 \mathrm{sec}$ though both the joints are independently driven.

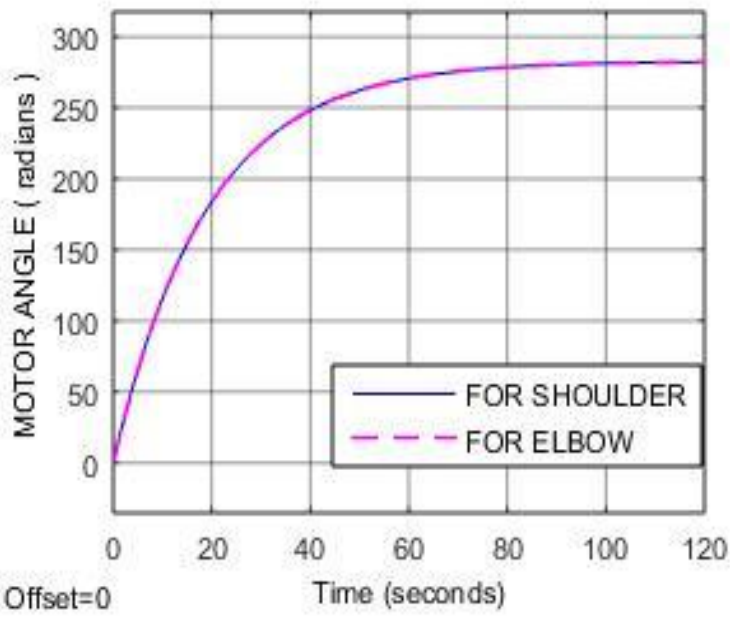

Figure 5 PMDC motor closed loop uncontrolled system without considering any disturbance torque

Mounting load on to the actuator, its torque gets fluctuates, as shoulder actuator will experience more disturbance due to the loading effect than that of elbow actuator as in Figure 6 , and thus angular position of the motor gets fluctuate as in Figure 7. Figure 8 shows the corresponding output joint angles of the links. Joint angles needs to show $\pi$ radians as an output for the input of 10 volts step signal, but loading effect made it to deviate from its desired position.

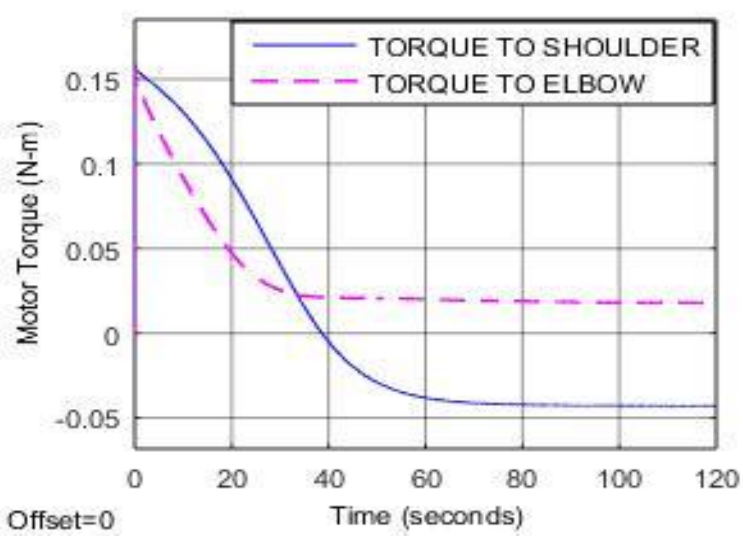

Figure 6 Motor torque disturbed due to the loading effect

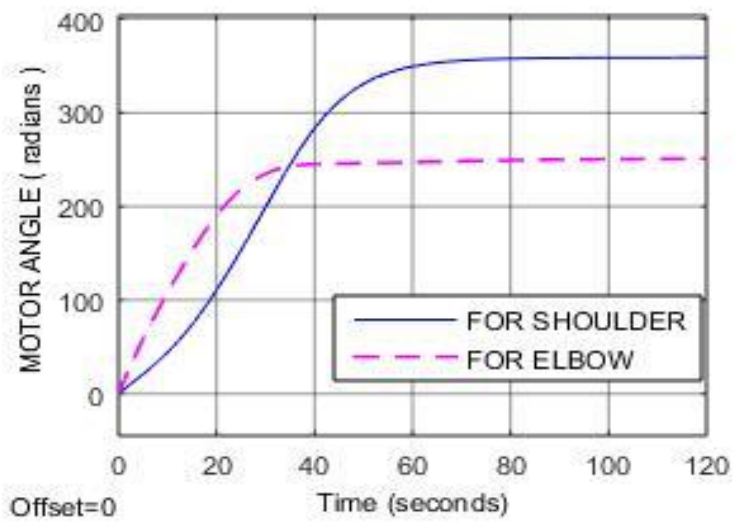

Figure 7 PMDC motor uncontrolled system with disturbance torque

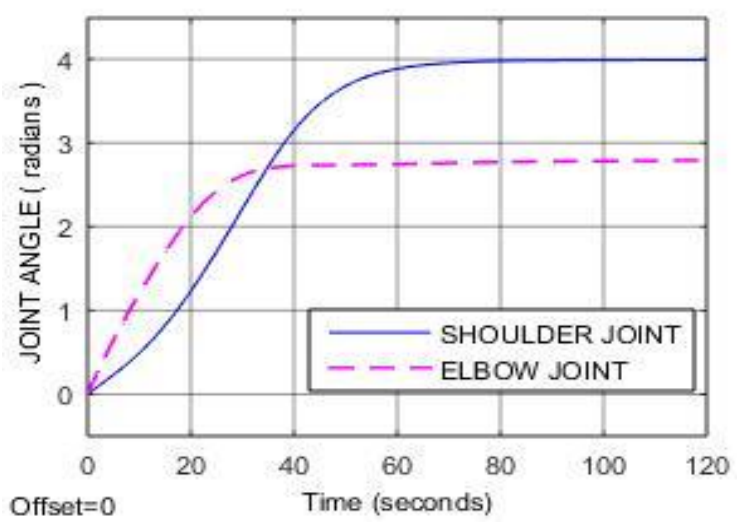

Figure 8 Joint angle position corresponding to the motor position

Controller is employed on the motor-side to control it by incorporating loading effects as disturbance to the motor torque. Classical PID will give very fast response of $50 \mathrm{msec}$ of settling time but it has very high peak overshoot as in Figure 9. PID tuned by GA 
will give comparatively much less peak overshoot though it has large settling time as compared to classical but overall performance has improved from the previous one, as in Figure 10 for the step input of 10 volts (corresponding desired position should be $\pi$ (3.141) radians). ( $K_{P}={ }_{153}, K_{I}={ }_{45}$ and $K_{D}={ }_{8}$.)

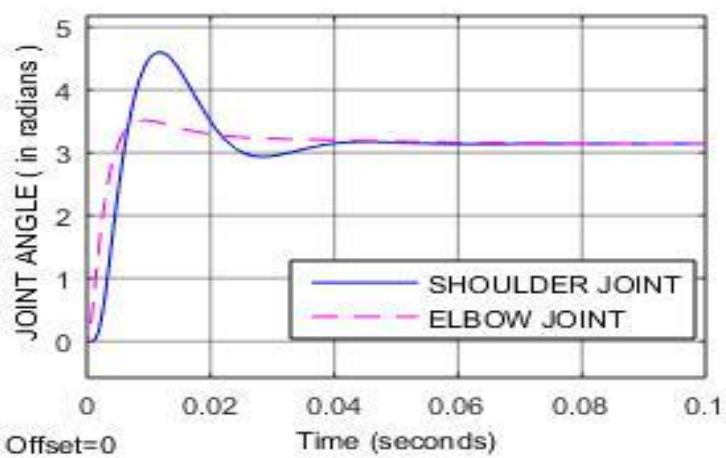

Figure 9 Classical PID response

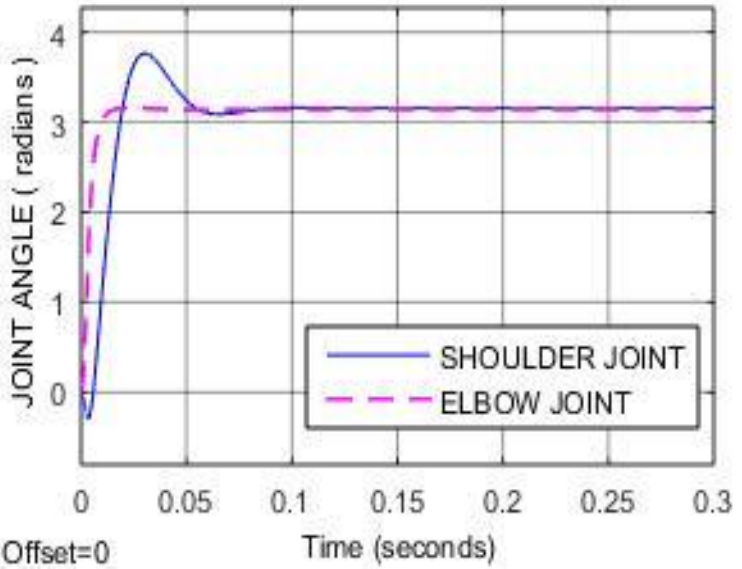

Figure 10 Response of PID tuned using GA

\section{Conclusion}

This paper encloses brief history of PID and its tuning techniques along with a glimpse of Genetic Algorithm. GA is used to tuned parameters of PID to control disturbance caused due to loading effect. Tremendous amount of work has already been done on PID and its tuning schemes. Though here this controlling technique is employed to show that by clubbing it with idea of motor-side control only (and allowing link side to interact freely with the environment) we can have efficient control of the system used for the slow motion applications. It is very easy computationally as well as analytically. As slow motion tracking is concern, we can always avoid the loading effect on motor due to the factors like environmental disturbances and uncertainties (on link side) by keeping the gear ratio as low as possible. It has some limitations like disturbances must be within limits and for slow motion tracking. But as long as system is under limits, this works amazingly and has very simple structure.

\section{Acknowledgment}

None.

\section{Conflicts of interest}

The authors have no conflicts of interest to declare.

\section{References}

[1] Güler S, Özgüler AB. Tracking and regulation control of a 2-DOF robot arm with unbalance. In international conference on methods and models in automation and robotics 2012 (pp. 280-5). IEEE.

[2] Saha S, Das S, Das S, Gupta A. A conformal mapping based fractional order approach for sub-optimal tuning of PID controllers with guaranteed dominant pole placement. Communications in Nonlinear Science and Numerical Simulation. 2012; 17(9):3628-42.

[3] Yu HC, Chen TC, Liu CS. Adaptive fuzzy logic proportional-integral-derivative control for a miniature autofocus voice coil motor actuator with retaining force. IEEE Transactions on Magnetics.2014; $50(11): 1-4$

[4] Han J. From PID to active disturbance rejection control. IEEE Transactions on Industrial Electronics. 2009;56(3):900-6.

[5] Spong MW, Hutchinson S, Vidyasagar M. Robot modeling and control. New York: Wiley; 2006.

[6] Samhouri M, Raoufi A, Surgenor B. Control of a pneumatic gantry robot for grinding: a neuro-fuzzy approach to PID tuning. In proceedings of IEEE conference on control applications 2005. (pp. 452-8). IEEE.

[7] Dumlu A, Erenturk K. Trajectory tracking control for a 3-DOF parallel manipulator using fractional-order control. IEEE Transactions on Industrial Electronics. 2014;61(7):3417-26.

[8] Cao YJ, Wu QH. Teaching genetic algorithm using MATLAB. International Journal of Electrical Engineering Education. 1999;36(2):139-53.

[9] Saad MS, Jamaluddin H, Darus IZ. Implementation of PID controller tuning using differential evolution and genetic algorithms. International Journal of Innovative Computing, Information and Control. 2012; 8(11): 7761-79.

[10] Wang QG, Zhang Z, Astrom KJ, Chek LS. Guaranteed dominant pole placement with PID controllers. Journal of Process Control. 2009;19(2):349-52.

[11] Mahfouz AA, Mohammed MK, Salem FA. Modeling, simulation and dynamics analysis issues of electric motor, for mechatronics applications, using different approaches and verification by MATLAB/simulink. International Journal of Intelligent Systems and Applications. 2013;5(5):39. 
Mayur Pateliya et al.

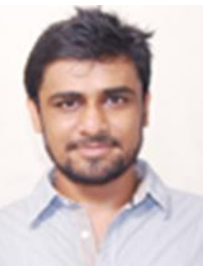

modeling and control of Robotics.

Email:mayurpateliya_3144611@nitkkr.ac.in
Mayur Pateliya was born in Bhavnagar, Gujarat, India in 1992. He has graduated from L.D. college of Engineering, Ahmedabad, Gujarat, India in 2013. Currently he is pursuing M.Tech from National Institute of Technology, Kurukshetra, Haryana, India. His current research interest is in

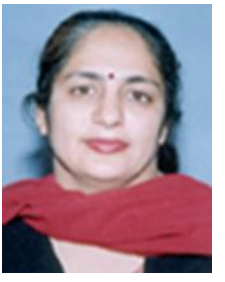

Jyoti Ohri has received her Ph.D from National Institute of Technology, Kurukshetra, Haryana, India in 2010. She is currently working as a Professor with National Institute of Technology, Kurukshetra, India. She has published more than 35 research publications in reputed journals and conferences. Her areas of current interest are Robust and adaptive control, intelligent control, optimization, Instrumentation and robotics. 